\title{
Fundamental aspects of electromigrative separation techniques
}

\author{
Carolin Huhn ${ }^{1}$ - Pablo A. Kler ${ }^{2}$
}

(C) Springer-Verlag Berlin Heidelberg 2016

Electromigrative separation techniques currently present an excellent option for the analysis of important analytes for various industrial and scientific application fields. A large number of different separation modes exist, which are based on different physicochemical properties (i.e., different separation mechanisms) of the analytes and working buffers. These allow development of efficient and specific methods for the targeted applications mostly using the same equipment. Tailoring selectivity is possible with the addition of secondary selectors acting by complexation, ion-pairing, or affinity binding among others. Sensitivity and selectivity can be addressed using various detection systems, including, most often optical, conductivity and mass spectrometric detection.

A clear benefit of electromigrative separation techniques is their comparatively low operation and maintenance costs, due to the low consumption of solvents

Published in the topical collection Fundamental Aspects of Electromigrative Separation Techniques with guest editors Carolin Huhn and Pablo A. Kler.

Carolin Huhn

carolin.huhn@uni-tuebingen.de

Pablo A. Kler

kler@cimec.unl.edu.ar

1 Institut für Physikalische und Theoretische Chemie der Universität Tübingen, Fachbereich Chemie, Auf der Morgenstelle 18, 72076 Tübingen, Germany

2 Centro de Investigación de Métodos Computacionales (CIMEC, UNL - CONICET), Colectora RN 168, Paraje El Pozo, Santa Fe S3000GLN, Argentina and other disposable materials, but also due to the robustness of the equipment, with few mobile parts. All these advantages have driven electromigrative separations to be among the most popular analytical techniques for portable and miniaturized devices for targeted and in-place analysis. In addition, the high ratio between analytical performance versus operational cost coerced the industry to develop high throughput and automated equipment for high numbers of samples, both in classic and miniaturized formats.

Finally, there is a last but major benefit of electromigrative separation techniques: in contrast to chromatographic techniques, a well validated collection of mathematical models exists that describes comprehensively all physicochemical processes taking place during electromigration. All these models are not only able to explain the analyte migration behavior and the performance of different separation mechanisms but they also enable the scientific community to understand and predict separation effects, mechanisms, and phenomena. It is especially the combination of well-chosen experimental designs and theoretical considerations that make electromigrative separation a prospering field of research, enabling novel applications in various fields such as pharmaceutical, biotechnological, and food science. In this topical collection, we thus focus on these fundamentals of electromigrative separation techniques on the one hand. Examples in this special issue are, e.g., unusual stacking phenomena, theory of affinity electrophoresis, and quantitative aspects of isotachophoresis. On the other hand, evolution of the techniques has to be supported and further developed by instrumental innovations. Two major topics are evolving recently: coupling electromigrative separation technique with mass spectrometric detection, and two-dimensional separations. The first enabled reaching high sensitivity 
and specificity to allow the users to deal with complex samples, particularly coming from biotechnological and biomedical applications such as the analysis of monoclonal antibodies and other complex bio-pharmaceutical drugs. In this topical collection, we present studies exploring new hyphenation approaches using dielectric barrier discharge ionization and surfactant-free micro-emulsion electrokinetic chromatography. Multidimensional separations with two electromigrative separation techniques such as capillary electrophoresis and isoelectric focusing are addressed with regard to chip- and valvebased interfaces including on-chip detection.

In the preceding paragraphs, we summarize the underlying philosophy of this topical collection: emphasizing that the systematic study of the fundamentals of electromigrative separation techniques and the development of new theoretical models together with instrumental innovations form the basis of exciting, new, and challenging analytical applications and problem-solving.

Therefore, we are pleased to present this topical collection for the issue of Analytical and Bioanalytical Chemistry in which we try to show the current dynamics of research on electromigrative separation techniques through the constant evolution of their fundamental understanding and the new instrumental developments, which continuously enhance the already amazing analytical capabilities of these techniques. We hope that the readers of $\mathrm{ABC}$ can enjoy this topical collection in the same way we did while preparing it. We thank all authors for submitting their contributions, the referees for their critical and constructive comments, and the editorial team of Analytical and Bioanalytical Chemistry for their highly professional collaboration and encouragement.

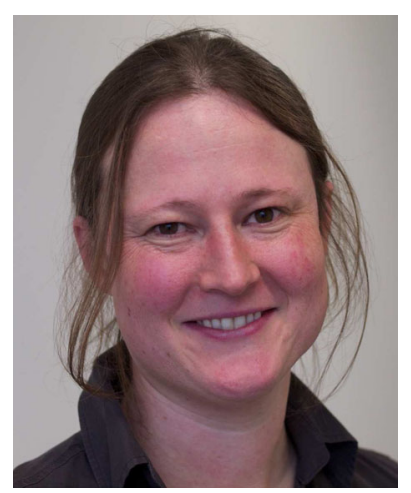

Carolin Huhn is a chemist by training and since 2013 Professor for Effect-based Environmental Analysis at the Eberhard Karls Universität Tübingen and speaker of the platform Environmental System Analysis at the university. Her research interests cover all aspects of analytical separation science with more than 10 years of experience in electromigrative separation techniques: instrumental development, fundamental studies, method development, and applications. Major focuses are electromigrative separation and enrichment techniques, including multidimensional separations with microfluidic elements and hyphenation to mass spectrometry as well as their application in forensics, pharmacy, clinics, and bio- and environmental analysis.

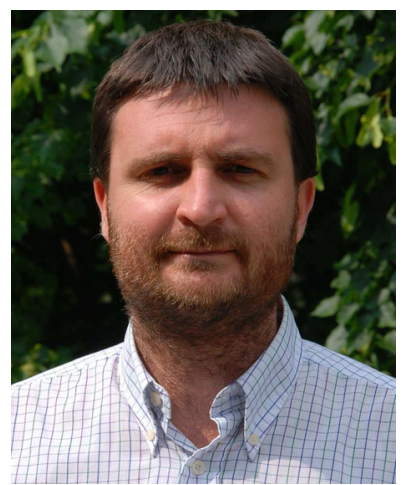

Pablo A. Kler is a research scientist at the Research Center for Computational Methods (CIMEC), at the Universidad Nacional del Litoral in Santa Fe, Argentina. He is also Assistant Professor at the Informatics System Engineering Department at the Universidad Tecnológica Nacional. He has been working since 2005 on modeling and simulations of microfluidics and transport phenomena at the microscale, with emphasis on electromigrative separation techniques for standard as well as for "Lab-on-a-chip" platforms. Currently he is developing computational tools for the optimization of different analytical techniques, including CE-MS and paper-based LFIA. 